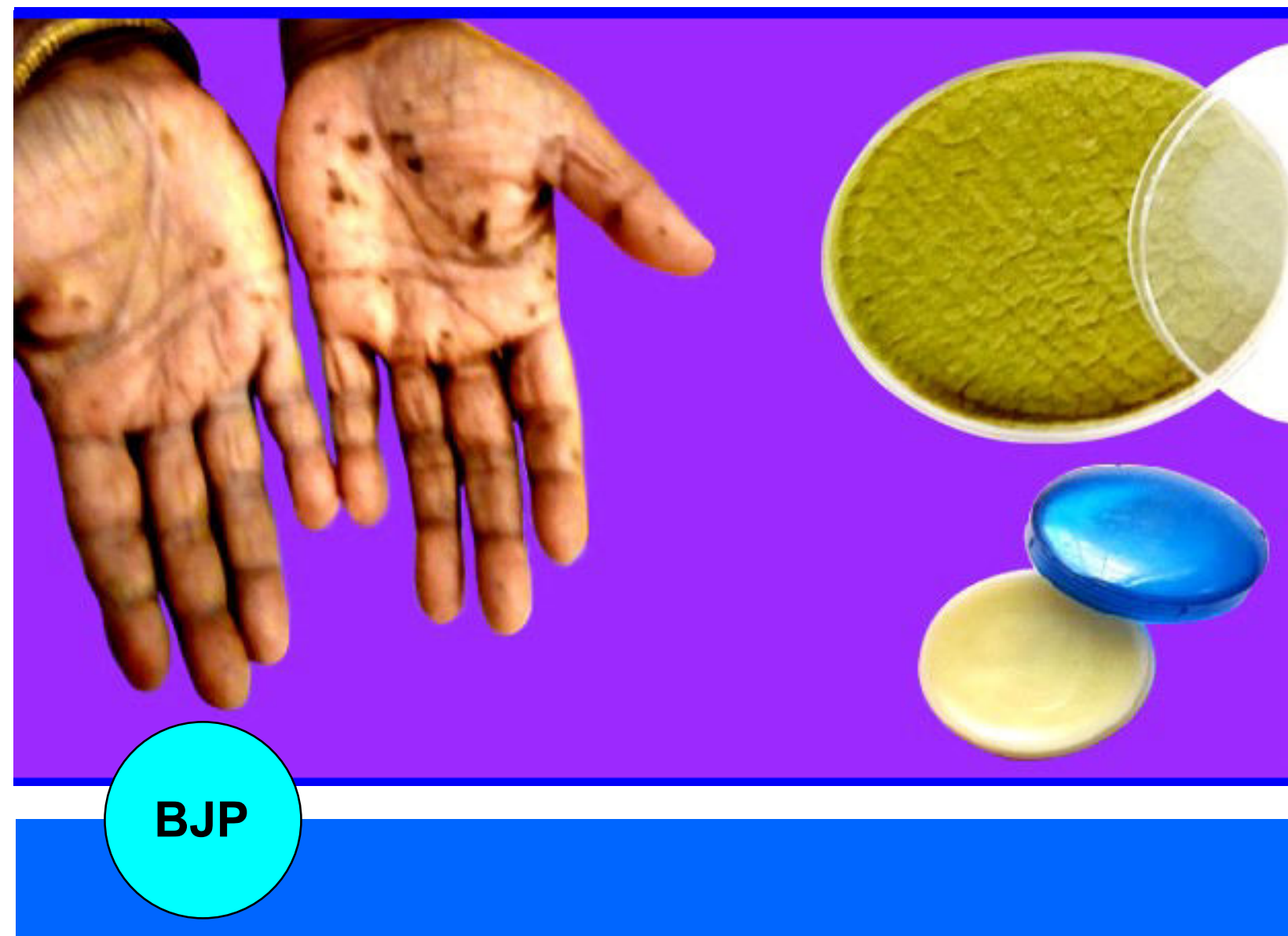

Bangladesh Journal of Pharmacology

Clinical Trial

Effects of tetracycline and clotrimazole ointments in the treatment of palmar arsenical keratosis 


\title{
Effects of tetracycline and clotrimazole ointments in the treatment of palmar arsenical keratosis
}

\author{
Asiya Ferdous ${ }^{1}$, Mir Misbahuddin ${ }^{1}$ and Abu Naser Ibne Sattar² \\ ${ }^{1}$ Division of Arsenic Research. Department of Pharmacology, Faculty of Basic Science and Paraclinical Science, \\ Bangabandhu Sheikh Mujib Medical University, Dhaka 1000, Bangladesh; 'Department of Microbiology and \\ Immunology, Faculty of Basic Science and Paraclinical Science, Bangabandhu Sheikh Mujib Medical University, \\ Dhaka 1000, Bangladesh.
}

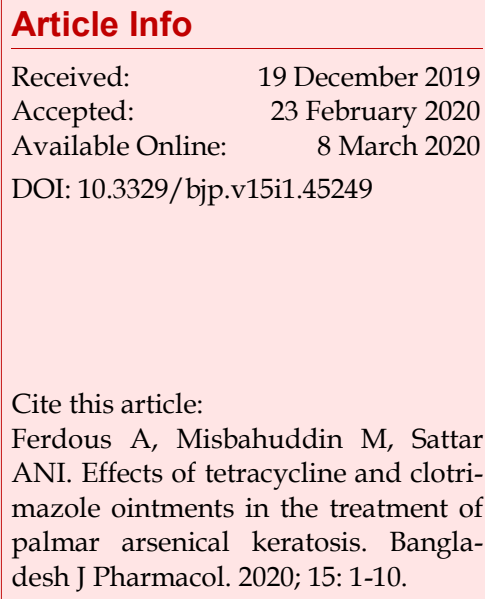

\section{Introduction}

Exposure to arsenic through contaminated groundwater is a major public health problem worldwide (Khan et al., 1997). Prolonged ingestion of arsenic above the safe level gives rise to a chronic health condition, termed as arsenicosis which is manifested usually by skin lesions, like- melanosis, leucomelanosis and keratosis, with or without involving the internal organs (Caussy, 2005). Arsenical keratosis, appearing on the palmar and plantar aspect of the hand and foot, is more troublesome for the patient as it affects the patient's socio-economically as well (Shahidullah et al., 2001; Hassan et al., 2005; de Luzuriaga, 2011; Safiuddin et al., 2011).

The cause of keratosis is not well-understood. Despite exposure to the same source of arsenic-contaminated groundwater, not all the members of the family get affected by arsenicosis. It had been found in certain studies that there is an alteration of normal skin flora in arsenicosis, where Aspergillus spp. and Enterobacter spp. were found to be significantly present on the palm of the patients with arsenical keratosis in comparison to the control population (Khalil et al., 2016; Moitra et al., 2018). But the relationship between such altered skin flora and the development of arsenical keratosis is not established or explored.

Moreover, the treatment of keratosis is not standardized. The most common therapeutic options lead to short-term improvement with recurrence after the stoppage of treatments. The oral formulations studied to treat keratosis are the antioxidants (Yerebakan et al., 2002; Son et al., 2008; Khandker et al., 2006), garlic oil (Misbahuddin et al., 2013), kala jeera oil (Bashar et al., 2014), spirulina, zinc (Misbahuddin et al., 2006; Rahman et al., 2006) and selenium (Momin et al., 2007; Krohn et 
al., 2016).

Topical treatment options, based mainly on keratolytic or water-retaining property, are salicylic acid (Islam et al., 2007), propylene glycol (Dina and Misbahuddin, 2010), neem (Ferdous and Misbahuddin, 2014), brinjal peel (Sarah, 2018) and cock's comb (Anny, 2018).

Reconstructive surgery with total excision of the keratotic skin followed by grafting is also used in cases of severe arsenical keratosis.

There is no such study where the effects of antimicrobials have been evaluated as the treatment of arsenical keratosis. So, the present study was designed to see the effects of topical antimicrobials on the altered skin flora (Aspergillus spp. and Enterobacter spp.) in patients with palmar arsenical keratosis.

\section{Materials and Methods \\ Place and duration of the study}

The study was conducted at three places: a) The Department of Pharmacology, Bangabandhu Sheikh Mujib Medical University, b) The Department of Microbiology and Immunology, Bangabandhu Sheikh Mujib Medical University, and c) Eruain Village of Kandirpara Union of Laksham Upazilla of Cumilla District. This study started in September 2017 and continued up to January 2019.

\section{Determination of sample size}

The present study was intended to propose or generate a hypothesis that the alteration of skin microbial flora may cause the development of keratosis. As there is no such work previously conducted, samples were taken as much as possible from those who fulfilled the selection criteria.

\section{Selection of arsenic-endemic area}

According to the Department of Public Health Engineering of the Government of Bangladesh, Cumilla is one of the worst arsenic-affected areas. After visiting multiple arsenic-affected endemic areas, Laksham, one of the Upazillas of Cumilla District was selected to be the study area as the local authority of the Upazilla as well as the patients found to be co-operative.

\section{Selection criteria}

In total, 38 patients with moderate to severe palmar arsenical keratosis were enrolled as per the inclusion and exclusion criteria of the study (Figure 1).

Inclusion criteria include a) Age: 18 to 60 years, b) drinking arsenic-contaminated water $(>50 \mu \mathrm{g} / \mathrm{L})$ for more than 6 months, c) patients with moderate to severe palmar arsenical keratosis, d) patients voluntarily agreed to participate, e) patients who did not receive topical application of any drug for the last three months, $\mathrm{f}$ ) patients who understood the instructions of applying drug and could apply the drug as per instructions.

Exclusion criteria include a) Age less than 18 years and more than 60 years, b) patients who received any treatment of arsenicosis within the last three months, c) patients with diagnosed skin diseases, like- atopic dermatitis and psoriasis (as per history obtained from patients), d) any diagnosed systemic disease, inflamamatory disease or infectious condition that affects the skin, like- diabetes mellitus, rheumatoid arthritis, systemic lupus erythematosus and hepatitis (as per history obtained from the patients).

\section{Allocation of the patients randomly into 4 groups}

Thirty-eight patients were randomized into 4 groups: Group 1: received tetracycline; Group 2: received clotrimazole; Group 3: received the combination of tetracycline and clotrimazole; Group 4: received placebo (no active ingredient)

Randomization and sequence generation were done using an online graph pad calculator. Four sets of random numbers were allocated as intervention (tetracycline, clotrimazole, the combination of tetracycline and clotrimazole) and placebo sequentially according to their ID number.

\section{Collection of samples}

The samples include drinking water, nail, skin swab and skin scraping.

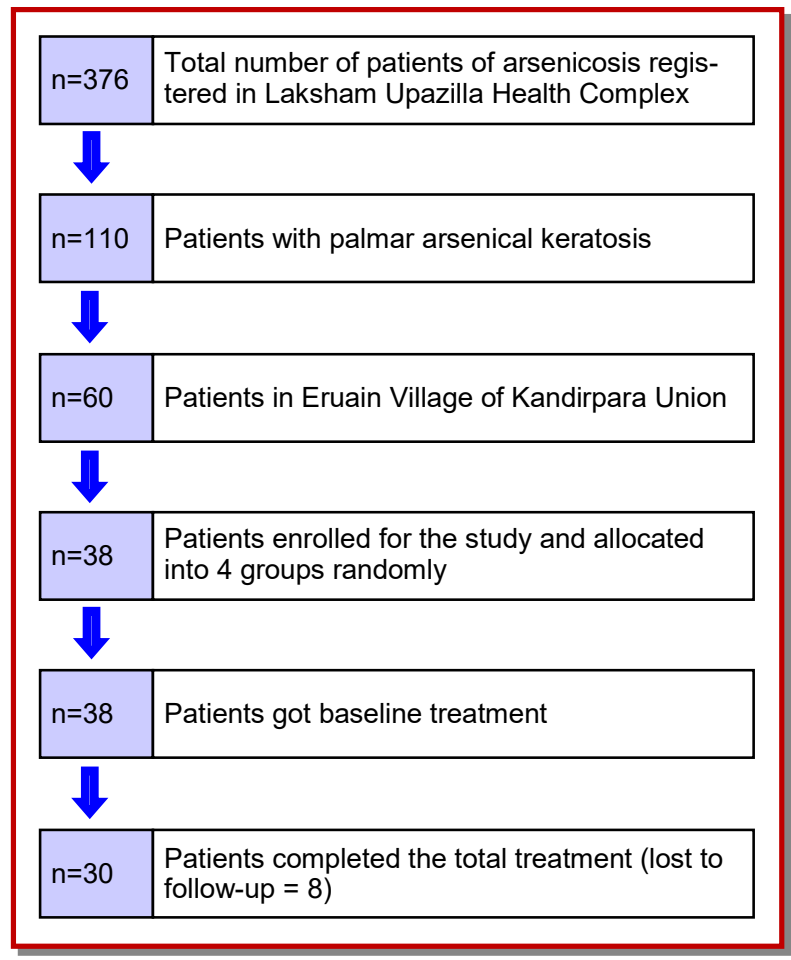

Figure 1: Steps of enrolling patients 


\section{Collection of water samples}

Patients were provided a $100 \mathrm{~mL}$ plastic container marked with an ID number containing 2-3 drops of nitric acid and requested to bring water from the tube well they use at present or used previously for drinking purposes. Then the samples were transported to the laboratory and stored at the refrigerator until analysis.

\section{Collection of nail samples}

Patients were requested to grow their nails. At first, they were advised to wash and dry their hands and nails before cutting the nails. They were provided a dry plastic bag marked with an ID number to collect nails from all their fingers. The nail samples were collected repeatedly until the amount became at least $200 \mathrm{mg}$. The collected samples were transported to the laboratory and stored at refrigerator until analysis.

\section{Collection of samples from the skin}

For the collection of skin swab and scraping samples, the screwed cap test tube containing nutrient broth media and petri dishes containing chromogenic agar media and potato dextrose agar media were taken to the field, keeping those in an icebox to stop the activity of the media during transportation.

Both hands were observed for keratotic lesions. The hand with more keratotic lesions was chosen for sample collection.

\section{Collection of skin scraping sample}

After washing the palm with $70 \%$ alcohol, skin scrapings were collected from the keratotic lesions with the help of a sterile surgical blade in a glass slide or container. By heating a wire loop in the flame of a candle until it became red, the wire loop was touched on the surface of the potato dextrose agar media containing petri dish to cool the wire loop to avoid killing of the microorganisms within the collected scraping samples and also to moist the wire loop with the media for easy collection of the dry scraping samples into petri dishes. After the collection of samples, petri dishes were sealed with micropore. The samples were kept for 7 days at room temperature for allowing the growth of the fungi.

\section{Collection of skin swab sample}

The skin of all the patients washed with $70 \%$ alcohol again. Then skin swabs were collected from each of the patients by rubbing the palm eight times by a sterile swab stick, moistened with a solution of nutrient broth. Then chromogenic agar media was inoculated by that swab stick. After collection of samples, petri dishes were sealed with micropore and transported to the microbiology laboratory and kept in the incubator for 18-24 hours (Jyoti et al., 2011; Khalil et al., 2016).

\section{Preparation of culture media}

Potato dextrose agar media, chromogenic media, MacConkey agar media, motility indole urease media were prepared, autoclaved and then cooled until the media solidified in the petri dish.

\section{Examination of fungal growth in culture plates}

The growth of the fungi appeared on day 3 in potato dextrose agar media containing petri dishes. No further growth was observed after day 7 .

Fungal growth was taken from petri dishes into the slides for microscopic examination.

\section{Examination of bacterial growth in culture plates}

The growth of the bacteria appeared in chromogenic agar media containing petri dishes after 18-24 hours incubation at $36^{\circ} \mathrm{C}$. The microorganisms were identified by observing the color of the colony (Samra et al., 1998).

As Enterobacter spp. and Klebsiella spp. both produce the same colored colony in chromogenic agar plates, these two were differentiated by doing motility indole urease test.

\section{Antimicrobial drug susceptibility test for isolated Aspergillus spp. and Enterobacter spp.}

Antifungal susceptibility test of Aspergillus spp. was performed against eleven antifungal drugs (clotrimazole, econazole, fluconazole, griseofulvin, itraconazole, ketoconazole, miconazole, nystatin, oxiconazole, terbinafine and tioconazole), whereas antibacterial susceptibility test of Enterobacter spp. was performed against eight antibacterial drugs (amoxicillin, cefixime, cefradine, cefuroxime, ciprofloxacin, co-trimoxazole, nitrofurantoin and tetracycline) by agar dilution method. The antimicrobial agents were obtained from the market.

\section{Agar dilution method}

Potato dextrose agar media was used for antifungal susceptibility test. MacConkey agar media was used for the antibacterial susceptibility test. The stock solution of antimicrobial agents, working solution of antimicrobial agents, antimicrobial drug supplemented media, and inoculum were prepared. After inoculating the organisms in the antifungal and antibacterial agent containing media, incubation was done. Then the growth of microorganisms (Provine and Hadley, 2000; Kuzucu et al., 2004; Razia et al., 2014) was observed. Growth in drug-containing media was compared with drug-free control media as follows: a) Growth same as control (score: 4); b) Slight decrease in growth (growth by approximately $75 \%$ of that control) (score: 3 ); c) Significant reduction in growth (growth approximately $50 \%$ of that control) (score: 2); d) Slight growth (growth approximately $25 \%$ of that control) (score: 1 ); and No growth (score: 0).

Effect of $p H$ on the growth of microorganisms in 


\section{presence of antimicrobials}

The steps to see the effect of $\mathrm{pH}$ on the growth of organisms: a) Preparation of drug supplemented $\mathrm{pH}$ adjusted ( $\mathrm{pH} 4-8$ ) media; b) inoculation of organisms; and c) observation of growth. The steps were repeated 5 times.

\section{Measurement of arsenical keratotic nodular size}

A slide caliper was used to take the measurements. Up to five keratotic lesions were measured in both hands. Both the length and breadth of each keratotic nodule were measured. After summing up all the nodular size in both hands, an average keratotic nodular size was calculated by dividing that with the number of lesions measured. This procedure was followed for each patient before initiating the study and after completion of 3 months of treatment.

\section{Preparation of ointment}

To make 1,000 g ointment, ingredients were used as follows: a) At first, stearyl alcohol and bee wax were melted together on a water bath; b) Then, white petrolatum was added and mixed well; c) $8 \mathrm{~mL}$ of $5 \%$ sodium hydroxide was added to make the $\mathrm{pH}$ of the ointment 8 (tested by litmus paper); d) Then the mixture was removed from the bath and stirred until the mixture became congealed.

\section{Distribution of medicines}

Tetracycline, clotrimazole, the combination of tetracycline and clotrimazole and placebo (ointment without any active ingredient) were given to the patients with moderate to severe palmar arsenical keratosis randomly. The patients were instructed to apply the ointment to the affected area of the palm by fingertip twice daily (in the morning and bedtime). The patients were asked to clean their hands before applying ointment. A printed sheet was given for checking the adherence by giving a tick mark at the appropriate place just after applying ointment. They were requested to bring the sheet on the next follow-up.

\section{Periodic monitoring of the patients}

The patients were visited by the researcher at the Eruain Community Clinic at two weeks interval. The keratotic nodular size was measured. Patients' perception of clinical improvement was asked. They were also asked to report adverse effects related to the use of ointment. Regular communication with the patient was maintained over the cell phone.

\section{Estimation of total arsenic}

The total arsenic level in the water and nail was estimated by the SDDC method using a spectrophotometer (UV-VIS spectrophotometer-1201, Shimadzu, Japan) at $525 \mathrm{~nm}$. The details of the method had already been described earlier (Bhuiyan et al., 2015).

\section{Statistical analysis}

Microsoft Office Excel was used to present the data as mean \pm SD. To compare the arsenical skin lesions size before and after treatment in each group, Wilcoxon matched-pairs signed-rank test was performed manually. The comparison between each of the treatment groups and placebo was done manually by MannWhitney U-test.

\section{Results}

Most of the patients enrolled were female with a malefemale ratio 1:4 (Table I). The mean $( \pm \mathrm{SD})$ age of the patients was $40.8 \pm 12.9$ years. The patients were exposed to arsenic through arsenic-contaminated drinking

\section{Table I}

\section{Characteristics of the patients}

\begin{tabular}{|l|r|}
\hline Parameters \\
\hline Gender & 6 \\
Male & 24 \\
Female & $40.8 \pm 12.9$ \\
Age (years) & $18.1 \pm 9.7$ \\
$\begin{array}{l}\text { Duration of exposure to arsenic through } \\
\text { contaminated drinking water (years) }\end{array}$ & $13.4 \pm 8.7$ \\
$\begin{array}{l}\text { Duration of development of keratosis } \\
\text { (years) }\end{array}$ & \\
$\begin{array}{l}\text { Concentration of arsenic in tube-well water } \\
(\mu g / L)\end{array}$ & $839.8 \pm 179.9$ \\
Concentration of arsenic in nail ( $\mu \mathrm{g} / \mathrm{g})$ & $13.2 \pm 12.8$ \\
\hline Data are presented as mean \pm SD & \\
\hline
\end{tabular}

\begin{tabular}{|l|}
\hline \multicolumn{2}{|c|}{ Table II } \\
\hline \multicolumn{2}{|c|}{ Palmar skin swab of the patients revealing } \\
the growth of bacteria and fungi \\
\hline \multicolumn{2}{|c|}{$\begin{array}{c}\text { No. of the positive } \\
\text { samples in skin swab }\end{array}$} \\
\hline Isolated bacteria (n=10) \\
Staphylococcus spp. \\
Enterobacter spp. \\
Klebsiella spp. \\
Proteus spp. \\
Pseudomonas spp. \\
Enterococcus spp. \\
Isolated fungi (n=15) \\
Aspergillus spp. \\
Dermatophytes spp. \\
Mucor spp. \\
Unidentified \\
Growth in chromogenic agar media for bacteria; Growth in potato \\
dextrose agar media for fungi \\
\hline
\end{tabular}




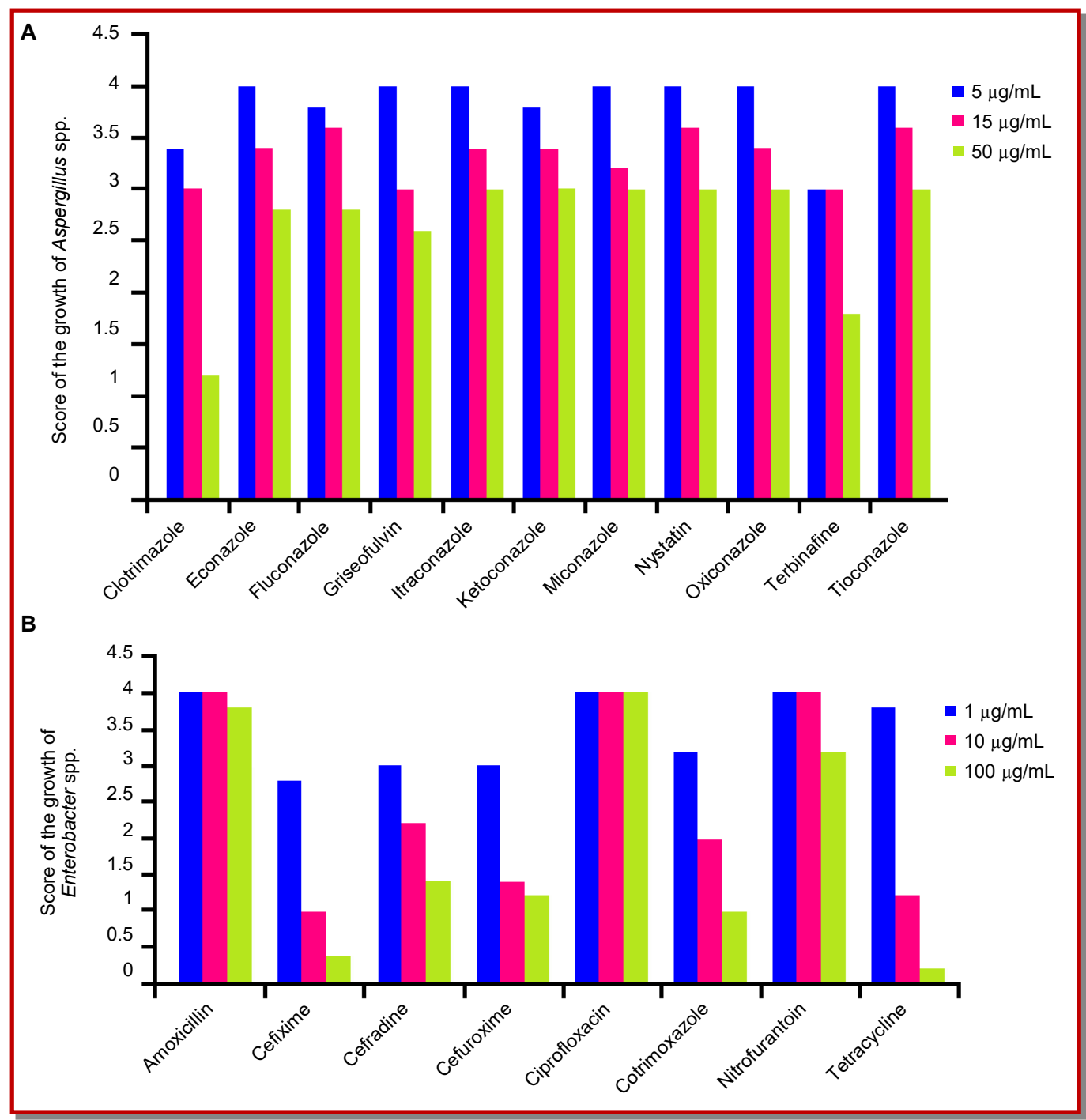

Figure 2: Diagram showing the effectiveness of different antifungals in inhibiting the growth of Aspergillus spp. at different concentrations in potato dextrose agar media (A); Diagram showing the effectiveness of antibacterials in inhibiting the growth of Enterobacter spp. at different concentrations in MacConkey agar media (B)

water for $18.1 \pm 9.7$ years and it took $13.4 \pm 8.7$ years to develop keratosis. The mean concentration of arsenic was $839.0 \pm 179.9 \mu \mathrm{g} / \mathrm{L}$ in drinking water and $13.2 \pm$ $12.8 \mu \mathrm{g} / \mathrm{g}$ in nail samples.

Table II shows that among the 15 skin scraping samples, 6 samples revealed the growth of Aspergilus spp. Gram-positive organism, Staphylococcus spp. was isolated from 9 out of 10 skin swab samples. Five types of Gram-negative organisms were found in the skin swab samples of which Enterobacter spp. was isolated from 3 samples.

Figure 2 shows each of the 11 antifungal drugs inhibited the growth of Aspergillus spp. The growth of inhibition was found to be increased with an increase in the concentration of antifungals. At $50 \mu \mathrm{g} / \mathrm{mL}$, the growth inhibition was found to be highest followed by terbinafine. Seven antibiotics out of eight inhibited the growth

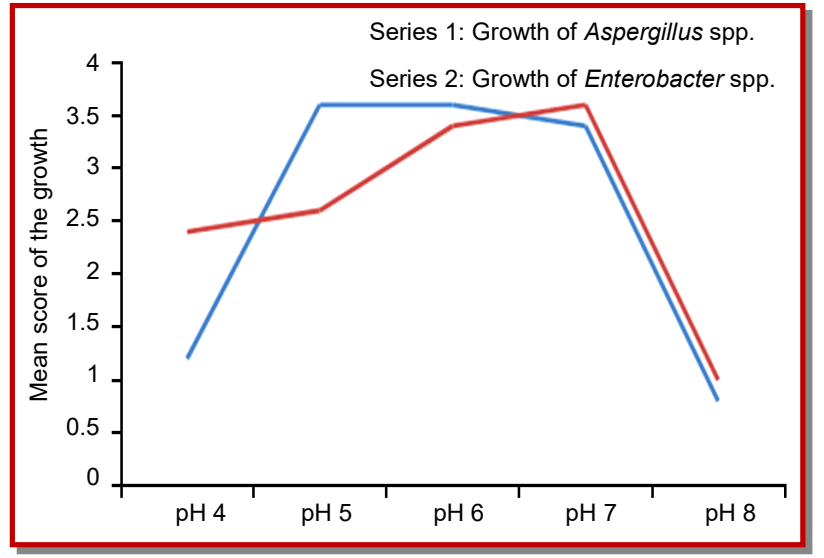

Figure 3: Diagram showing the inhibition of the growth of Aspergillus spp. and Enterobacter spp. by clotrimazole $(30 \mu \mathrm{g} / \mathrm{mL})$ and tetracycline $(50 \mu \mathrm{g} / \mathrm{mL})$ at $\mathrm{pH}$-adjusted potato dextrose agar media and MacConkey agar media respectively 


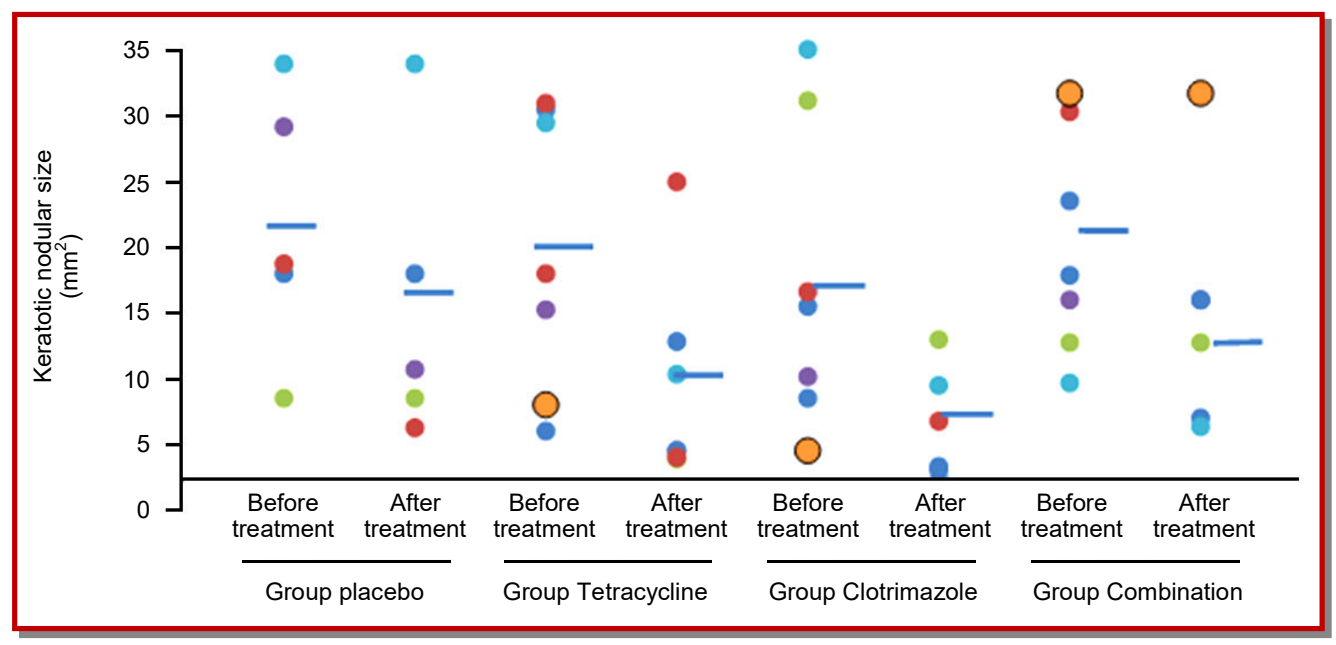

Figure 4: Changes of keratotic nodular size before and after treatment

of Enterobacter spp. and the inhibition was found to be increased gradually at higher concentrations. The growth of inhibition at $100 \mu \mathrm{g} / \mathrm{mL}$ was highest with tetracycline. Ciprofloxacin did not inhibit the growth even at the highest concentration.

The $\mathrm{pH}$ of the palmar surface of the hand (mean \pm SD) was found to be close in the arsenic-exposed participants $(8.0 \pm 0.2)$ and arsenicosis $(8.0 \pm 0.2)$, whereas the $\mathrm{pH}$ was lower in the group of healthy volunteers $(7.5 \pm$ $0.3)$. This difference was found statistically significant.

There was $\mathrm{pH}$-dependent inhibition of the growth of Aspergillus spp. in presence of clotrimazole $(30 \mu \mathrm{g} / \mathrm{mL})$ in potato dextrose agar media. Figure 3 shows the maximum inhibition of the growth of Aspergillus spp. at $\mathrm{pH} 8$, followed by $\mathrm{pH} 4$ and 5. In the case of Enterobacter spp., similar $\mathrm{pH}$-dependent inhibition of the growth was observed in the presence of tetracycline $(50 \mu \mathrm{g} /$ $\mathrm{mL}$ ) in MacConkey agar media. The maximum inhibition of the growth was found at $\mathrm{pH} 4$ and 8 .

The size of the keratotic nodule before and after the intervention was $21.7 \pm 10.1 \mathrm{~mm}^{2}$ and $15.5 \pm 11.2 \mathrm{~mm}^{2}$ respectively in the placebo group (Figure 4) and the improvement was found statistically insignificant with $\mathrm{p}$ value of 0.11 . In the tetracycline group, the size of the keratotic nodule before and after the intervention was $18.3 \pm 10.7 \mathrm{~mm}^{2}$ and $8.1 \pm 7.9 \mathrm{~mm}^{2}$ respectively. The improvement was found statistically significant ( $p$ value 0.01 ) with the percentage of keratosis size reduction 66.1. The mean $\pm \mathrm{SD}$ of the keratotic nodule size was $17.4 \pm 11.6 \mathrm{~mm}^{2}$ and $5.5 \pm 4.4 \mathrm{~mm}^{2}$ before and after treatment with clotrimazole ointment for 3 months. The percentage of keratosis size reduction was 61.1 and the clinical improvement was found statistically significant with $\mathrm{p}$ value 0.02 . The keratotic nodule size in the combination group was $20.3 \pm 8.6 \mathrm{~mm}^{2}$ and $12.8 \pm$ $10.2 \mathrm{~mm}^{2}$ before and after intervention respectively, which was found not to be significant statistically ( $p$ value 0.07 ). Each of the three groups of intervention (tetracycline, clotrimazole and combination) was compared with the placebo group to see the superiority among the interventions and not found statistically significant.

The adherence of the patients toward intervention was assessed and found $94.3 \pm 6.0 \%$ (tetracycline), $91.3 \pm$ $9.1 \%$ (clotrimazole) and $97.5 \pm 4.0 \%$ (a combination of tetracycline and clotrimazole), whereas in the placebotreated patients, the adherence was $83 \pm 14.8 \%$. Only three patients complained about adverse effects which were not significant and subsided spontaneously.

\section{Discussion}

This is the first report showing the effect of topical antimicrobials in the treatment of palmar arsenical keratosis. The patients of palmar arsenical keratosis were treated with the topical application of antibacterial and antifungal agents for 3 months. Either tetracycline or clotrimazole was found to be effective in the reduction of the size of the keratosis. However, the combination of tetracycline and clotrimazole did not show any improvement in comparison to the placebo. The adherence of the patients toward intervention and placebo was satisfactory with no remarkable adverse effect.

There are several studies characterizing the normal flora of the skin in both culture-dependent and independent techniques. Studies exploring microbial flora in healthy skin found Microcooci spp. (Pillsburry and Rebell, 1951; Aly and Maibach, 1977), Coryne-bacterium spp. (Pillsburry and Rebell, 1951; Aly and Maibach, 1977; Grice et al., 2009), Nocardia spp. (Pillsburry and Rebell, 1951), Propionibacterium spp.(Pillsburry and Rebell, 1951; Grice et al., 2009), coagulase-negative Staphylococci spp. (Aly and Maibach, 1977; Grice et al., 2009) as normal skin Gram-positive bacterial flora. On the other hand, Klebsiella spp., Proteus spp., Acinetobacter spp., Enterobacter spp. and E.coli were the predominant 
Gram-negative organisms isolated (Aly and Maibach, 1977). Candida spp. and Pityrosporon spp. or Malassezia spp. (Pillsburry and Rebell, 1951; Roth and James, 1988; Paulino et al., 2006) were reported as the most common fungus in the skin of healthy individuals Two studies conducted at this laboratory showed S. epidermidis and Bacillus spp. as normal bacterial flora (Khalil et al., 2016) and species of Aspergillus, Dermatophytes, Mucor and Fusarium as normal fungal flora (Moitra et al., 2018).

The normal skin flora can be altered by a variety of factors which can be either endogenous or exogenous environmental influences (Roth and James, 1988). Limited studies had been conducted to see the alterations of normal skin flora in arsenicosis. One of the diagnostic features of arsenicosis is skin lesions. These skin lesions, arsenic itself and its metabolites may play an important role in determining the composition of the skin flora in arsenicosis.

In a study, skin swab samples collected from 3 anatomical sites (arm, finger web and upper chest) were analyzed by culture techniques and later identification of microorganisms was done by biochemical test or PCR method. The results revealed an increased Gramnegative bacterial load in arsenicosis in comparison to the control population (Jyoti et al., 2011). Enterobacter spp. was found to be present in the palmar surface of the skin in patients with arsenical keratosis significantly when compared with arsenic-exposed individuals and healthy volunteers. A significant number of the samples revealed the presence of Enterobacter spp. in patients with palmar arsenical keratosis (Khalil et al., 2016). On the other hand, Aspergillus spp. was found to be predominant fungus in the palmar surface in comparison to arsenic-exposed and healthy individuals (Moitra et al., 2018). The present study also explored and found the presence of Aspergillus spp. and Enterobacter spp. as a skin flora in patients with moderate to severe palmar arsenical keratosis. The variation in the percentage of the isolated organism in these studies may be due to variation in the number of samples obtained, techniques by which the samples were collected and transported, techniques used for isolation and identification of the organisms (culture-dependent or molecular techniques), the media used in case of culture-dependent studies.

Whether this altered flora of the palmar surface of the skin in arsenicosis is responsible for the development of keratosis, the present study was conducted to see the effect of antimicrobials targeting that altered flora in clinical improvement. To choose the antimicrobials against Aspergillus spp. and Enterobacter spp., the antimicrobial susceptibility test was performed.

There are several studies evaluating antimicrobial susceptibility against microorganisms. The performance of semisolid agar antifungal susceptibility method
(Kuzucu et al., 2004), CLSI broth microdilution method (Tokarzewski et al., 2012; Baidee et al., 2012), EUCAST method (Lass-florl et al., 2008), disk diffusion method and diffusion-dilution method (Tokarzewski et al., 2012) and E-test method (Baidee et al., 2012) were assessed for a range of anti-fungal agents (amphotericin B, amphotericin B lipid complex, itraconazole, voriconazole, posaconazole, caspofungin, terbinafine, tioconazole, ketoconazole, clotrimazole, miconazole and nystatin) and found different agents to be effective against different species of Aspergillus.

The antimicrobial susceptibility against Enterobacter differs widely as there are diverse species within the genus. E. sakazakii and E. agglomerans were found susceptible to ampicillin, cephalothin, and cefoxitin, to which E. cloacae and E. aerogenes were resistant (Muytjens and Repe, 1986). 100\% sensitivity of isolated Enterobacter spp. to imipenem and $70 \%$ to amikacin was reported by the disk diffusion method (Sharmin et al., 2009). Another study found reduced sensitivity to a second-generation cephalosporin (Rangaiahagari et al., 2013). Expanded-spectrum $\beta$-lactamase producing $E$. cloacae revealed statistically significant higher resistance to cefotaxime, ceftazidime, aztreonam, piperacillin, tetracycline, and cotrimoxazole than non-producers (Wang et al., 2017).

The present study assessed the activity of 11 antifungal and 8 antibacterial drugs against Aspergillus spp. and Enterobacter spp. isolated from the palmar surface of the skin of the patients with arsenical keratosis by agar dilution method and found clotrimazole and tetracycline to be the most effective ones. The susceptibility pattern of antimicrobials in the present study differs from other studies due to the variation in the species of Aspergillus and Enterobacter investigated, test methods, media used, size of the inoculum, range of concentrations of drugs and assessment techniques.

Stoppage of drinking arsenic-contaminated water is the first step toward the management of arsenicosis. In addition, emphasis should be given on the provision of a diet rich in protein and vitamins. Intake of plenty of green leafy vegetables is also advisable. Previously, both enteral and topical approaches had been used to treat palmar arsenical keratosis.

Retinoid (Son et al., 2008), vitamin E (Verret et al., 2005), combination of vitamin A, C and E (Khandker et al., 2006), folic acid (Gamble et al., 2007), zinc and spirulina (Misbahuddin et al., 2006; Rahman et al., 2006; Misbahuddin and Afrin, 2013), selenium (Momin et al., 2007; Krohn et al., 2016), garlic oil (Misbahuddin et al., 2013), kala jeera oil (Bashar et al., 2014) and probiotics (Rashid et al., 2014) were tested in several studies to treat arsenicosis. These oral approaches to treatment options are not sign-specific and required a longer time to relieve. 
Keratosis present in the palm and sole can be treated by topical application as well. Salicylic acid (Islam et al., 2007) with or without urea, propylene glycol (Dina and Misbahuddin, 2010), neem (Ferdous and Misbahuddin, 2014), brinjal peel (Sarah, 2018), cock's comb (Anny and Misbahuddin, 2019) were some of the agents studied to treat keratosis. No remarkable adverse effects were reported due to these topical treatments, except itching and burning sensation. Most of these local treatment options either have keratolytic (Islam et al., 2007; Dina and Misbahuddin, 2010; Sarah, 2018) or water-retaining properties that regulate the normal desquamation process of the skin (Anny and Misbahuddin, 2019). As this is the first study where antimicrobials were used as a treatment option for keratosis, some factors, likeconcentration of the drug in the ointment, $\mathrm{pH}$ of the ointment and adherence toward treatment were assessed.

As the present study showed that there was better inhibition of the growth of Enterobacter spp. and Aspergillus spp. by $100 \mu \mathrm{g} / \mathrm{mL}$ tetracycline and $50 \mu \mathrm{g} / \mathrm{mL}$ clotrimazole respectively, to prepare 1,000 g ointment, $100 \mathrm{mg} / \mathrm{mL}$ tetracycline and $50 \mathrm{mg} / \mathrm{mL}$ clotrimazole had been used.

In the present study, the interventions were given for 3 months. Adherence in each of the intervention and placebo groups was assessed and found satisfactory. The earlier studies using the local approach of treatment reported the clinical improvement either by measuring percentage reduction of keratotic nodular size or by assessing perception scores. In the present study, both the breadth and length of 1-5 keratotic nodules of both palms were measured. Very few adverse effects were reported in the present study.

The percentage reduction of keratotic nodular size after 3 months of treatment was 66.1 and 61.1 in tetracycline and clotrimazole groups of patients, which was found statistically significant. The small number of patients (58 ) in each of the 4 groups and the duration of the treatment should be considered for better clinical improvement. The interventions (tetracycline, clotrimazole and combination of tetracycline and clotrimazole) were not found statistically superior in comparison to placebo.

\section{Conclusion}

In vitro study shows that tetracycline or clotrimazole was found to be effective against Enterobacter spp. and Aspergilus spp. respectively in $\mathrm{pH}$-dependent manner. In patients of arsenicosis, the clinical improvements after 3 months of treatment with tetracycline or clotrimazole ointments were found statistically significant. No intervention (tetracycline, clotrimazole or combination of tetracycline and clotrimazole) was found statistically superior in comparison to placebo in reducing palmar arsenical keratotic nodular size.

\section{Ethical Issue}

The research was conducted as per the protocol approved on January 21, 2018 by the institutional Review Board of Bangabandhu Sheikh Mujib Medical University (Registration number: BSMMU/2018/924). Informed written consent was taken from each participant with strict maintenance of their confidentiality. Every step was taken to look after and monitor the patients.

\section{Conflict of Interest}

Authors declare no conflict of interest.

\section{References}

Aly R, Maibach HI. Aerobic microbial flora of intertriginous skin. Appl Environ Microbiol. 1977; 33: 97-100.

Anny F, Misbahuddin M. Effect of cock's comb extract in the treatment of palmar arsenical keratosis. Bangladesh J Pharmacol. 2019; 14: 87-92.

Baidee P, Alborzi A, Moeini M, Haddadi P, Farshad S, Japoni A, Ziyacyan M. Antifungal susceptibility of the Aspergillus species by Etest and CLSI reference method. Arch Iran Med. 2012; 15: 429-32.

Bashar T, Misbahuddin M, Hossain MA. A double-blind, randomized, placebo-controlled trial to evaluate the effect of Nigella sativa on palmar arsenical keratosis patients. Bangladesh J Pharmacol. 2014; 9: 15-21.

Bhuiyan HA, Tshering K, Misbahuddin M. Estimation of arsenic in nail using silver diethyldithiocarbamate method. Bangladesh J Pharmacol. 2015; 10: 513-17.

Caussy D. A field guide for detection, management and surveillance of arsenicosis cases. New Delhi, WHO Regional Office for South-East Asia, 2005.

de Luzuriaga AMR, Ahsan H, Shea CR. Arsenical keratoses in Bangladesh: Update and prevention strategies. Dermatologic Clinics. 2011; 29: 45-51.

Dina AN, Misbahuddin M. Randomized double-blind trial to evaluate the effectiveness of topical administration of propylene glycol in arsenical palmer keratosis. Bangladesh J Pharmacol. 2010; 5: 98-102.

Ferdoush J, Misbahuddin M. Effect of ethanol extract of leaves of Azadirachta indica on palmar arsenical keratosis: A singleblind trial. Bangladesh J Pharmacol. 2014; 9: 279-83.

Gamble MV, Liu X, Slavkovich V, Pilsner JR, Llievski V, Factor -Litvak P, Levy D, Alam S, Islam M, Parvez F, Ahsan H, Graziano JH. Folic acid supplementation lowers blood arsenic. Am J Clin Nutr. 2007; 86: 1202-09.

Grice EA, Kong HH, Conlan S, Deming CB, Davis J, Young AC, Bouffard GG, Blakesley RW, Murray PR, Green ED, Turner ML, Segre JA. Topographical and temporal diversity of the human skin microbiome. Science 2009; 324: 1190-92. 
Hassan MM, Atkins PJ, Dunn CE. Social implications of arsenic poisoning in Bangladesh. Soc Sci Med. 2005; 61: 2201-11.

Islam AZM, Misbahuddin M, Sikder S, Biswas AK, Islam Z, Hadiuzzaman, Khandakar S, Mahmud I, Ahmed SA. Randomized controlled trial to evaluate the effectiveness of topical use of salicylic acid for treatment of keratosis in arsenicosis patients. In: Applied Research on Arsenic in Bangladesh, World Health Organization (Dhaka, Bangladesh), Directorate General of Health Services, Government of Bangladesh, 2007.

Jyoti A, Bonny TS, Hossain MA. Arsenic poisoning alters the composition of skin microbial flora of human. Res J Microbiol. 2011; 6: 524-33.

Khalil NB, Misbahuddin M, Sattar ANI. Pattern of skin bacteria in palmar arsenical keratosis. Bangladesh J Pharmacol. 2016; 11: 940-43.

Khan AW, Ahmad SA, Sayed MMSU, Hadi SA, Khan MH, Jalil MA, Ahmed R, Faruquee MH. Arsenic contamination in ground water and its effects on human health with particular reference to Bangladesh. J Prev Soc Med. 1997; 16: 6573.

Khandker S, Dey RK, Islam AZMM, Ahmad SA, Mahmud IA. Arsenic-safe drinking water and anti-oxidants for the management of arsenicosis patients. Bangladesh J Pharmacol. 2006; 1: 42-50.

Krohn MR, Raqib R, Akhtar E, Vandenberg A, Smits JEG. A high-selenium lentil dietary intervention in Bangladesh to counteract arsenic toxicity: Study protocol for a randomized controlled trial. Trials 2016; 17: 1-8.

Kuzucu C, Rapino B, McDermott L, Hadley S. Comparison of the semisolid agar antifungal susceptibility test with the NCCLS M38-P broth microdilution test for screening of filamentous fungi. J Clin Microbiol. 2004; 42: 1224-27.

Lass-Florl C, Mayr A, Perkhofer S, Hinterberger G, Hausdorfer J, Speth J, Fille M. Activities of antifungal agents against yeasts and filamentous fungi: Assessment according to the methodology of the European Committee on Antimicrobial Susceptibility Testing. Antimicrob Agents Chemother. 2008; 52: 3637-41.

Misbahuddin M, Islam AZMM, Khandker S, Ifthaker-AlMahmud, Islam N, Anjumanara. Efficacy of spirulina extract plus zinc in patients of chronic arsenic poisoning: A randomized placebo-controlled study. Clin Toxicol. 2006; 44: $135-41$.

Misbahuddin M, Afrin M. Effect of spirulina on the levels of zinc, vitamin $\mathrm{E}$ and linoleic acid in the palm skin extracts of people with prolonged exposure to arsenic. Bangladesh J Pharmacol. 2013; 8: 84-91.

Misbahuddin M, Bashar T, Hossain MA. Effectiveness of garlic oil in the treatment of arsenical palmar keratosis. Bangladesh J Pharmacol. 2013; 8: 22-27.

Moitra P, Misbahuddin M, Sattar ANI. Pattern of skin fungi in palmar arsenical keratosis. Bangladesh J Pharmacol. 2018; 13: $1-9$.

Momin A, Ali SK, Haque MM. Randomized placebo-controlled trial to evaluate the effectiveness of selenium in the treatment of arsenicosis patients. In: Applied Research on Arsenic in Bangladesh, World Health Organization (Dhaka, Bangladesh), Directorate General of Health Services, Government of Bangladesh, 2007.

Muytjens HL, Repe JR. Comparative in vitro susceptibilities of eight Enterobacter species with special reference to Enterobacter sakazakii. Antimicrob Agents Chemother. 1986; 29: 36770.

Paulino LC, Tseng C, Strober BE, Blaser MJ. Molecular analysis of fungal microbiota in samples from healthy human skin and psoriatic lesions. J Clin Microbiol. 2006; 44: 2933-41.

Pillsbury DM, Rebell G. The bacterial flora of the skin: Factors influencing the growth of resident and transient organisms. J Investig Dermatol. 1952; 18: 173-86.

Provine H, Hadley S. Preliminary evaluation of semisolid agar antifungal susceptibility test for yeasts and molds. J Clin Microbiol. 2000; 38: 537-42.

Rahman MH, Sikder MS, Islam AZMM, Wahab MA. Spirulina as food supplement is effective in arsenicosis. J Pakistan Assoc Dermatologists. 2006; 16: 69-75.

Rangaiahagari A, Uwizeyimana1 JP, Nyirabanzi1 J, Ngoga E, Wane J. Antibiotic sensitivity patterns of Enterobacteriaceae isolated at King Faisal Hospital, Kigali: A three years study. Rwanda Med J. 2013; 70: 11-4.

Rashid N, Misbahuddin M, Choudhry ZK, Saleh AA, Sattar ANI. The colony count of Escherichia coli in the stool of palmar arsenical keratosis following probiotics supplementation. Bangladesh J Pharmacol. 2014; 9: 176-81.

Razia S, Anwar S, Miah MRA, Nahar N, Barua R. Evaluation of semisolid agar method for antifungal susceptibility test of $T$. rubrum. Bangabandhu Sheikh Mujib Med Univ J. 2014; 7: 114.

Roth RR, James WD. Microbial ecology of the skin. Annu Rev Microbiol. 1988; 42: 441-64.

Safiuddin MD, Shirazi SM, Yusoff S. Arsenic contamination of groundwater in Bangladesh: A review. Int J Phys Sci. 2011; 6: $6791-800$

Samra Z, Heifetz M, Talmor J, Bain E, Bahar J. Evaluation of use of a new chromogenic agar in detection of urinary tract pathogens. J Clin Microbiol. 1998; 36: 990-94.

Sarah QS. Effects of Solanum magnum $L$ peel extract in the treatment of arsenical skin lesion. MD thesis, Bangabandhu Sheikh Mujib Medical University, 2018.

Shahidullah M, Sadir AM, Hossain MZ. Arsenicosis in Bangladesh. Bangladesh J Derma Venerol Leprol. 2001; 18: 45-52.

Sharmin S, Alamgir F, Fahmida, Saleh AA. Antimicrobial sensitivity pattern of uropathogens in children. Bangladesh J Med Microbiol. 2009; 3: 18-22.

Son SB, Song HJ, Son SW. Successful treatment of palmoplantar arsenical keratosis with a combination of keratolytics and low-dose acitretin. Clin Exp Dermatol. 2008; 33: 202-04.

Tokarzewski S, Ziółkowska G, Nowakiewicz A. Susceptibility testing of Aspergillus niger strains isolated from poultry to antifungal drugs: A comparative study of the disk diffusion, broth microdilution (M 38-A) and Etest methods. Pol J Vet Sci. 2012; 15: 125-33. 
Verret WJ, Chen Y, Ahmed A, Islam T, Parvez F, Kibriya MG, Graziano JH, Ahsan H. A randomized, double-blind, placebo -controlled trial evaluating the effects of vitamin $\mathrm{E}$ and selenium on arsenic-induced skin lesions in Bangladesh. J Occup Environ Med. 2005; 47: 1026-35.

Wang S, Xiao S, Gu F, Tang J, Guo X, Ni Y, Qu J, Han L.
Antimicrobial susceptibility and molecular epidemiology of clinical Enterobacter cloacae bloodstream isolates in Shanghai, China. PLoS One. 2017; 12: 1-12.

Yerebakan Ö, Ermis O, Yilmaz E, Basaran E. Treatment of arsenical keratosis and Bowen's disease with acitretin. Int J Derma. 2002; 41: 84-87. 